\begin{tabular}{c} 
International Journal of Engineering \& Technology, $7(3.22)(2018) 27-31$ \\
International Journal of Engineering \& Technology \\
SPC \\
Website: www.sciencepubco.com/index.php/IJET \\
Research paper \\
\hline
\end{tabular}

\title{
Ambiverts Potentially Have Better Orienting Response than Extraverts in Visual Oddball Paradigm of Event Related Potential
}

\author{
Nasir Yusoff*, Yan Shan Tai, Saidah Napisah Muhammad, Faruque Reza \\ Department of Neurosciences, School of Medical Sciences, Health Campus, Universiti Sains Malaysia, 16150 Kubang Kerian, Ke- \\ lantan, Malaysia \\ *Corresponding author E-mail: nasirff@usm.my
}

\begin{abstract}
Background: Differences in personality may indicate dissimilarity in the process of cognition. Objectives: To investigate and compare the neural substrate of P300 component evoked between ambiverts and extraverts in visual oddball paradigm of Event Related Potential study. Methods: Forty undergraduate medical students from Universiti Sains Malaysia (USM) were recruited and screened for personality by using USM Personality Inventory (USMaP-i). In the Event Related Potential (ERP) session, participants (N=19 ambiverts and 20 extraverts) completed a visual oddball paradigm in counterbalanced order. Results: Mann Whitney Test showed that, compared to ambiverts, the extraverts showed diminished P300 amplitude at the Fz electrode, but not at other electrodes. Conclusion: Ambiverts might have better orienting response than extraverts.
\end{abstract}

Keywords: Ambiversion, Extraversion, P300, Personality, Visual oddball

\section{Introduction}

The cognitive profiles of different personality types have garnered much interest in the advent of machines such as electroencephalography (EEG) and functional magnetic resonance imaging (fMRI). Among them, the extraversion-introversion continuum of the Big Five model is the most stable personality trait across a person's lifespan. ${ }^{[1]}$ More commonly understood as an indication of social adaptability, ${ }^{[2]}$ high levels of extraversion is associated with a preference for interpersonal interaction, excitement, impulsiveness, outspokenness, and popularity. ${ }^{[3],[4]}$

In his theory, Eysenck posited that one's level of cortical arousal explains their degree of extraversion. ${ }^{[5]}$ Extraverts have chronically lower levels of cortical arousal than introverts, thus leading them to seek stimulation from the external environment to achieve and maintain optimal cortical arousal. ${ }^{[6]}$ However, both extraverts and introverts are 'extreme scorers' on both ends of the extraversion scale and as such, most studies on these personality types are not reflective of the general population, whose personality fall between the two extremes. ${ }^{[7]}$

Ambiverts are neither introverts nor extraverts, but possess the features of introversion and extraversion which manifest in different situation. ${ }^{[8]}$ They are reasonably contented with social interaction, but can also appreciate time alone, away from crowds. In comparison, ambiverts have a different cognitive profile as evidenced by a lower cognitive impairment risk ${ }^{[9]}$ and higher IQ scores ${ }^{[10]}$ than both extraverts and introverts. Despite that fact that ambiverts represent the majority of the human population, only few researches compared the neurophysiological correlates of ambiverts to that of highly introverted and extroverted individuals. [11], [12], [13]
In personality research, the P300 ERP component is commonly investigated in relation to extraversion, and remains a popular non-invasive measure of attention, decision making, and memory or context updating. ${ }^{[14],[15],[16]}$ It is a large, phase-locked positive deflection that commonly occurs around $250 \mathrm{~ms}$ to $900 \mathrm{~ms}$ after presentation of a stimulus. ${ }^{[17]}$ In visual and auditory settings, extraverts produced smaller P300 amplitudes than introverts. ${ }^{\text {18], [19] }}$ Similar findings were obtained in studies of emotion processing, where extraverts with lower baseline arousal levels also produced smaller P300 amplitude. ${ }^{[15],[20]}$ Other studies by Beauducel et al. ${ }^{[21]}$, Gurrera et al. ${ }^{[22]}$, Gurrera et al. ${ }^{[23]}$ and Philipova ${ }^{[24]}$ however reported P300 amplitude to be positively correlated with extraversion levels whereas Lindin et al. ${ }^{[25]}$ and Ortiz et al. ${ }^{[26]}$ found no difference in P300 amplitude.

Extraverts and introverts react differently. It is reported that extraverts have faster/shorter motor response ${ }^{[27]}$ but slower thought processing speed ${ }^{[11]}$ compared to introverts. In another study, warning stimulus (that provided future information) induced larger P300 amplitudes in introverts than in extraverts, when compared to critical stimulus (that provided closure). In addition, P300 latency was also influenced by task demands. ${ }^{[11],[28]}$ The effort invested in tasks also differed by personality type based on the situational demands of the experiments ${ }^{[18]}$ as introverts allocated more sustained attention in vigilant tasks than extraverts. ${ }^{[29]}$

As demonstrated, most studies compared extreme scorers on the extraversion scale, but little light has been shed on how extreme scorers compare with the intermediate subgroup that better characterise the general population, i.e. ambiverts. In a proceeding originated from the current study, Yusoff et al. ${ }^{[30]}$ proposed that both personality types share similar cognition processing as measured by an EEG auditory oddball task where participants counted only the low tones; there were non-significant differences in P300 amplitudes and latencies between extraverts and ambiverts. In a simi- 
lar study, Georgiev et al. ${ }^{[7]}$ found instead that ambiverts produced greater P300 amplitudes than introverts and extraverts. However, different task demands revealed different findings. Georgiev et al. ${ }^{[7]}$ found that in comparison to introverts, extraverts and ambiverts produced larger P300 amplitudes in a passive auditory paradigm. In conditions requiring behavioural responses to the tones, Georgiev et al. ${ }^{[7]}$ found that extraverts exhibited the largest P300 amplitude.

Based on above background, thus, the current study sought to further investigate the P300 component of ambiverts by comparing the ambiverts' and extraverts' P300 responses to visual stimuli in unimodal oddball paradigms. It is hypothesised that there would be a significant difference in P300 amplitudes between ambiverts and extraverts in visual oddball paradigms.

\section{Methodology}

\subsection{Subjects}

Participants were 40 undergraduate medical students from a public university in Malaysia (Universiti Sains Malaysia, Health Campus). Twenty ambiverts and 20 extroverts were recruited, as determined by their responses on the USM Personality Inventory (USMaP-i). This study obtained ethical clearance from the Human Ethical Committee of Universiti Sains Malaysia (Reference number: USMKK/PPP/JEPeM [267.2.(7)]. They reported normal or corrected-to-normal vision, had no lifetime history of a major medial disorder (neurological, hepatic, or cardiovascular), no history of affective disorder, and no history of using psychiatric medication.

\subsection{Tools/Measure}

\subsubsection{The Universiti Sains Malaysia Personality Inventory (USMaP-i)}

The Universiti Sains Malaysia Personality Inventory (USMaP-i) was used in personality screening to classify respondents into different trait of personality - extravert and ambivert. ${ }^{[31]}$ The inventory was developed based on the Big-Five personality factors (i.e. Neuroticism, Extraversion, Openness to Experience, Agreeableness, and Conscientiousness), aimed to cater to sensitivity of population culture and value among Malaysian. The full version of the USMaP-i consists of 66-items with rating scales from 0 (very inaccurate) to 4 (very accurate). We only used the Extraversion properties (12 items from the 66 items) and using the following cut-off to determine the personality traits- extravert (scored of 33 to 48 ) and ambivert (scored of 17 to 32). In the current study, the internal consistency of the Extraversion sub-scale was excellent (Cronbach's alpha value of 0.82 ), indicated by 82 respondents.

\subsubsection{Event Related Potential (ERP)}

Event-related potential (ERP) is the electrical brain potential, indicated by the electroencephalogram motion in human brain, in relation to a definable sensory, cognitive, or motor event. It entails a series of peaks and troughs known as ERP components, measuring electrical potentials produced by the ion flow of extracellular fluid across cell membranes and the connection of neurons through neurotransmitter. ${ }^{[32]}$ Event related potential (ERP) indicates the characteristic of high temporal resolution due to the monitoring of brain electrical activity with high precision. Accordingly, ERP is said as valuable technique for observing different electrophysiological components that represent the various cognitive stages in relation to theories of perception and attention, ${ }^{[32]}$ as well as a potential tool in order to identify the electrophysiological components that represent the onset of cognitive dysfunction. ${ }^{[17]}$ Please refer to the section of procedure for the ERP recording.

\subsection{Procedure}

After providing written informed consent, the participants provided demographic details, and undergoing Event Related Potential session that was held in The Magnetoencephalography and Clinical Event Related Potential Laboratory, Hospital of Universiti Sains Malaysia. Participants completed visual oddball paradigms in a counterbalanced order.

\subsubsection{Visual oddball paradigm}

The visual oddball session consisted 240 trials. Prior to each trial, a fixation cross ('+') appeared for $300 \mathrm{~ms}$. Each trial presented a single stimulus at random for $1500 \mathrm{~ms}$; the stimulus could either be the standard stimulus, 'O', which was present in 180 trials (presenting about $75 \%$ of the trials), or the target stimulus, ' $\mathrm{X}$ ', which was present in 60 trials (presenting about $25 \%$ of the trials). The diagram of the oddball paradigm is shown in Figure 1 below. Participants pressed ' 1 ' on the keypad when they saw 'O' and '2' when they saw ' $\mathrm{X}$ '. All stimuli were $100 \mathrm{~mm} \times 100 \mathrm{~mm}$ in size and were white against a black background.

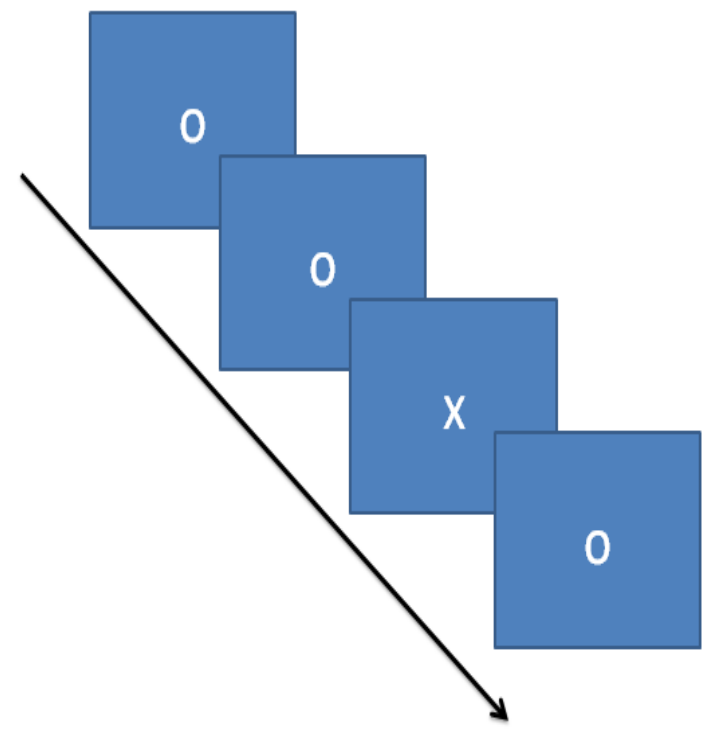

Fig.1: Schematic illustration of the oddball paradigm in Event Related Potential procedure (Press 1 for ' 0 ' and press 2 for ' $\mathrm{X}$ ')

\subsubsection{Event Related Potential (ERP) Recording}

In the recording chamber, the participant was positioned $80 \mathrm{~cm}$ in front of a 22" LCD screen monitor. A 128 HydroCel Geodesic Sensor Net (HCGSN) was applied symmetrically on the participant's head according to the standard 10-20 international electrode placement system. Net impedance was kept under $50 \mathrm{k} \Omega$ and the data was digitised at $250 \mathrm{~Hz}$. For the visual task, EEG data was recorded from electrodes $\mathrm{Fz}, \mathrm{Cz}, \mathrm{Pz}, \mathrm{C} 3, \mathrm{C} 4, \mathrm{~T} 5, \mathrm{~T} 6, \mathrm{O} 1$, and O2. The recording was completed using the EGI Net Station 5.3 software.

\subsubsection{EEG Pre-processing}

The EEG data was subjected to $0.30-30.00 \mathrm{~Hz}$ bandpass filter and a $50 \mathrm{~Hz}$ Notch filter. EEG for correct responses during each session was averaged separately, and the ERP waveforms were phased locked to $100 \mathrm{~ms}$ before and $1000 \mathrm{~ms}$ after stimulus onset. For each EEG data file, ocular and movement artefacts were detected with sliding windows of $80 \mathrm{~ms}$ and removed automatically, followed by bad channel replacement and averaging, after which the data was converted into the 10-20 montage and subjected to baseline correction. Subsequently, individual EEG data files were combined and averaged together. Statistical extraction was per- 
formed by the same software, and comprised of all amplitudes and latencies at the aforementioned electrode sites for target and standard tones.

Following the extraction of ERP component, data was further analysed using Statistical Package for Social Sciences (SPSS) version 24 (SPSS Inc. Chicago, Illinois). Since data did not fit up the requirement of parametric test, thus, nonparametric test (i.e. Mann Whitney Test) was undertaken to analysis the raw EEG data, which is to determine the significant difference of P300 ERP component between ambivert and extravert, in standard and target stimuli.

\section{Results}

\subsection{Sociodemographic Data}

After excluding an excessively noisy dataset, the final sample size was 19 ambiverts ( 8 males) and 20 extraverts (12 males). The mean age $( \pm \mathrm{SD})$ for the ambiversion group was $22.1( \pm 1.15)$ years and the mean age for the extraversion group $( \pm$ SD) was 22.7 ( \pm 1.41$)$ years. As the data showed non-normality, a MannWhitney $\mathrm{U}$ test was employed and revealed no significant age difference between the groups, $p>.05$. Mean personality scores on the USMaP-i $( \pm \mathrm{SD})$ for the ambiversion group and extraversion group were $28.95( \pm 2.97)$ and $38.45( \pm 3.87)$, respectively. A summary of the relevant sociodemographic data is shown in Table 1.

The peak voltage between $300 \mathrm{~ms}$ and $600 \mathrm{~ms}$ after stimulus onset was regarded as the P300 component. Subsequent analyses employed non-parametric tests (Mann-Whitney U Tests) as the data was not distributed normally.

Table 1: Socio-demographic profile

\begin{tabular}{|l|c|c|}
\hline Variables & Ambiverts & Extraverts \\
\hline Gender (Male / Female) & $8 / 11$ & $12 / 8$ \\
\hline Age (mean years \pm SD) & $22 \pm 1.15$ & $22.70 \pm 1.41$ \\
\hline USMaP-i score (mean \pm SD) & $28.95 \pm 2.97$ & $38.45 \pm 3.87$ \\
\hline Handedness (right / left) & $18 / 1$ & $16 / 4$ \\
\hline Race (Malay /Chinese/Indian) & $8 / 8 / 3$ & $9 / 9 / 2$ \\
\hline
\end{tabular}

\subsection{ERP Data for Visual Oddball Paradigm}

At most electrode sites, there were no significant differences in P300 amplitudes between both extraverts and intraverts in the target tone condition and in the standard tone condition, most $p \mathrm{~s}$ $>.05$. For target stimulus, P300 amplitude was significantly greater at the Fz site for the ambiversion group (mean $=5.41, \mathrm{SD}=3.60$ ) as compared to the extraversion group (mean $=2.49, \mathrm{SD}=1.46$ ), $Z=-3.37, P=.001$. For standard stimulus, P300 amplitude was significantly greater at the $\mathrm{Fz}$ site as well for the ambiversion group (mean $=3.02, \mathrm{SD}=1.78$ ) as compared to the extraversion group (mean $=1.86, \mathrm{SD}=1.86$ ), $Z=-2.47, P=.013$. These findings were depicted in Figures 2 and 3, respectively.

For the P300 latency, there were no significant differences between ambiverts and extraverts, at all electrodes.
8

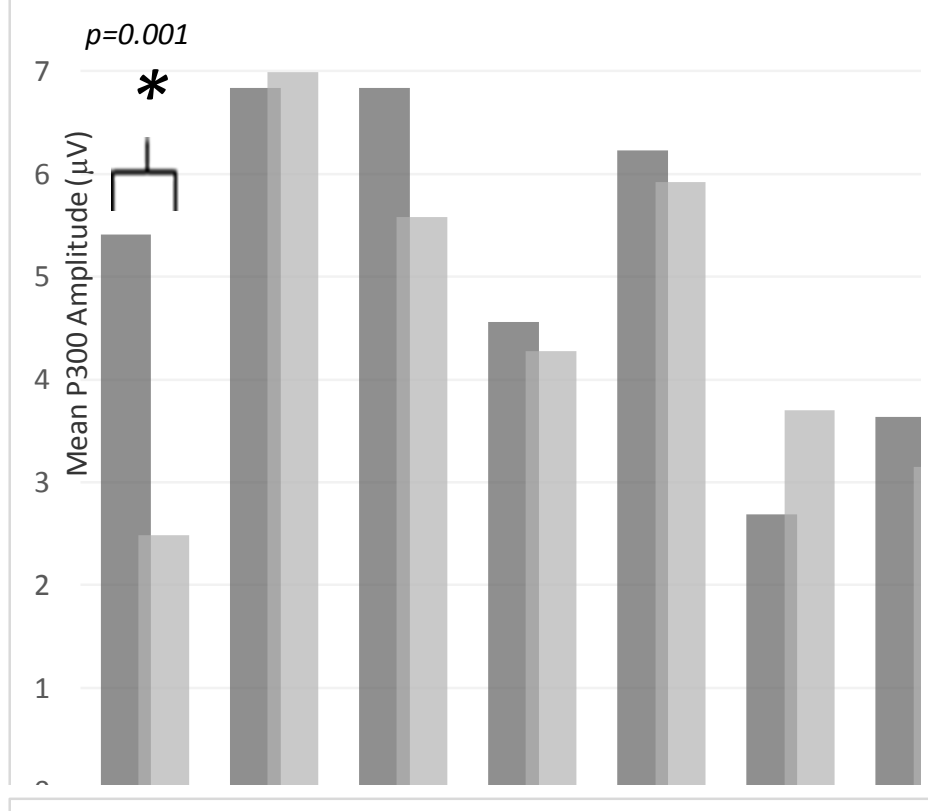

6

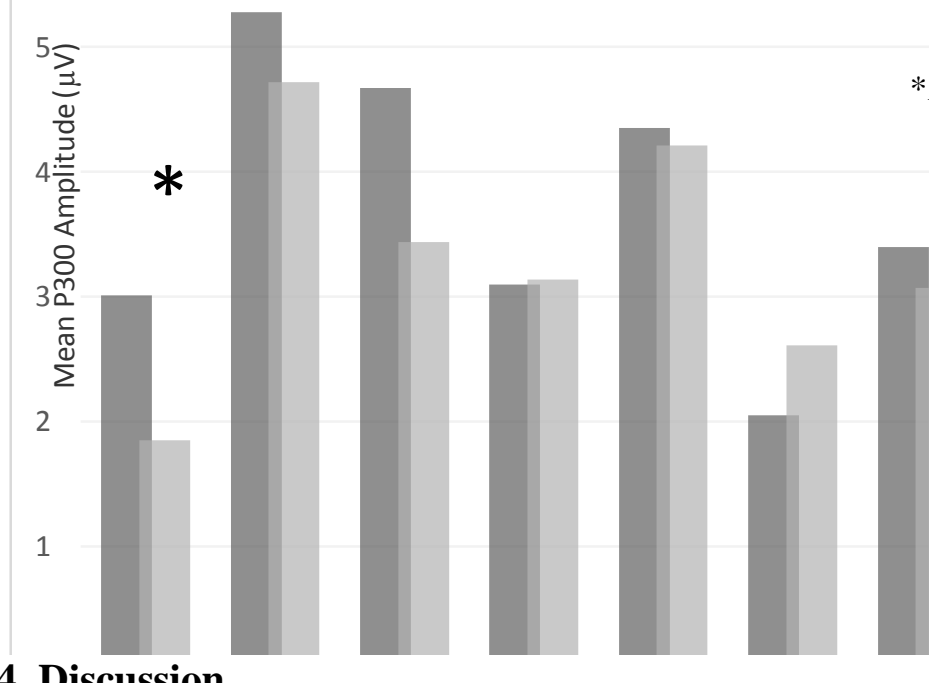

4. Discussion

The present study investigated the P300 component evoked in ambiverts and extraverts in response to visual stimuli in singlemodality oddball tasks. The P300 amplitudes was found to be significantly higher at electrode $\mathrm{Fz}$ among ambiverts as compared to extraverts for both standard and target stimuli. Meanwhile, the latencies of P300 were similar across groups and conditions. 


\subsection{P300 in the Visual Oddball Paradigm}

P300 amplitude has been shown to reflect allocation of attention, memory-updating and other cognitive processes whereas P300 latency reflects the time taken to evaluate or classify a stimulus before a behavioural response is generated. ${ }^{[33],[34]}$ At large, the current study showed no significant difference in P300 latencies for both personality types in all conditions, showing that ambiverts and extraverts took as long to evaluate the stimuli.

Past studies found that P300 amplitude was more pronounced among introverts as compared to extroverts, due to different baseline cortical arousal. ${ }^{[18],[35]}$ As ambiverts represent the intermediate between the two extremes, P300 amplitudes differences were expected. Brocke et al. ${ }^{[18]}$ compared the P300 component elicited in extraverts and introverts, and found the greatest difference at the Fz electrode site where introverts showed a higher amplitude than extraverts. It appears that by virtue of being 'in the middle', the P300 component elicited by ambiverts in the current study resembled that of introverts and extroverts. It is worth noting that the P300 amplitude did not differ at other electrodes sites aside from electrode $\mathrm{Fz}$, but not at the other midline electrodes $(\mathrm{Cz}$ and $\mathrm{Pz}$ ) which typically recorded the greatest P300 amplitudes over the centro-parietal region. ${ }^{[15]}$ While the $\mathrm{P} 3$ a subcomponent cannot easily be delineated from $\mathrm{P} 3 \mathrm{~b}$ in a classical oddball paradigms, its frontal distribution suggest that the difference in Fz P300 amplitude in the current study might point toward different degrees of automatic attentional capture by stimuli, ${ }^{[36], ~[37] ~ o t h e r w i s e ~ k n o w n ~}$ as the orienting response. ${ }^{[15]}$ This is different from the P300 evoked at the centro-parietal regions (P3b) which reflected voluntary, sustained attention toward a stimulus. ${ }^{[15]}$ Thus the current findings revealed that ambiverts might have a stronger orienting response than extroverts, but both personality groups were equally matched at voluntary attentional resource allocation. ${ }^{[15]}$

This interpretation is also built on the spontaneous EEG data obtained by Brocke et al. ${ }^{[18]}$ which recorded increasingly larger Alpha 1 power $(7.5 \mathrm{~Hz}-10 \mathrm{~Hz})$ for extroverts throughout their study, whereas Alpha 1 power remained consistent for introverts. This meant that over time extraverts generally became less aroused, which modulated attention levels afforded to a task. ${ }^{[15]}$ ${ }^{[38]}$ This could have resulted in the extraverts' smaller P300 amplitudes in the frontal region in the current study. This is especially so when extraverts complete monotonous tasks associated with low arousal levels, ${ }^{[39],[40]}$ such as the classical oddball paradigm employed in this study. Similarly, Von Gehlen et al. ${ }^{[41]}$ showed that introverts were more able to focus in such monotonous tasks or in quiet conditions, whereas extraverts thrive in noisy environments or when completing complex tasks. Thus being 'part introvert and part extravert', the ambiverts in the current study could focus better than the extraverts at the oddball task.

Reduced P300 amplitude at the Fz electrode site in extraverts could also suggest that mind-wandering or task-unrelated thoughts (TUTs) occurred more frequently among extraverts than ambiverts. Barron et al. ${ }^{[42]}$ found that higher frequencies of TUT were related to diminished P3a and P3b amplitudes. ${ }^{[43]}$ This showed that mind wandering decoupled the subject's attention from the external environment and redirected it to an internal train of thought, which dampens the brain's responsiveness to external stimuli. ${ }^{[42]}$ While current findings only revealed differences at the Fz electrode, this could be because ambiverts shared enough similarities with extraverts for them to have comparable neurocognitive profiles even during TUTs.

In an fMRI study, Christoff et al. ${ }^{[44]}$ also found that mindwandering mostly activated the default network regions in the medial prefrontal regions, which might explain why the differences between extraverts and ambiverts in the current study was most significant at the frontal region. The relatively under-aroused extraverts were also more likely than ambiverts to recruit the default network which underlies mind-wandering and TUTs, ${ }^{[45]}$ thus hampering P300 amplitude.

\subsection{Limitations and Strengths}

The current study is limited by the simplicity of the experimental paradigms involved, which could not substantiate the ideas explored in the discussion section well enough. Tentatively, the current study proposed that ambiverts and extraverts differed in their orienting response in response to visual stimulus. This suggestion should be investigated in future studies by employing threestimulus oddball tasks which are capable of delineating frontal and parietal P300. Including tasks with increasing levels of complexity or difficulty could also shed more light on the neurocognitive profiles of ambiverts and extraverts. Additionally, future studies should also take into account other psychological and biological variables that could potentially confound the P300 component. ${ }^{[46]}$

\section{Conclusions}

Few studies investigated the neurocognitive profile of ambiverts despite them representing the largest personality group. Using unimodal oddball tasks to investigate the P300 component, the current study revealed that both ambiverts and extraverts largely demonstrated similar attentional and memory-updating processes in response to visual stimuli. Importantly however was that ambiverts might have a stronger orienting response in comparison to extraverts in response to visual stimulus.

\section{Acknowledgments}

This study was supported by the Universiti Sains Malaysia (Research University Grant - 1001/PPSP/812188).

\section{References}

[1] P. Milojev, C. Sibley, The stability of adult personality varies across age: Evidence from a two-year longitudinal sample of adult New Zealanders, J Res Pers 51 (2014) 29-37

[2] M. Zuckerman, Psychobiology of personality, Cambridge University Press, New York, 1991.

[3] P.T. Costa Jr, T. A. Widiger, Personality disorders and the fivefactor model of personality, American Psychological Association, Washington, 1994.

[4] H.J. Eysenck, S.B.G Eysenck, Manual of the Eysenck Personality Questionnaire (junior and adult), University of London Press, London, 1975.

[5] H.J. Eysenck, Dimensions of personality: 16, 5 or 3-Criteria for a taxonomic paradigm, Pers Indiv Differ 12(8) (1991) 773-790.

[6] R.G. Geen, Preferred stimulation levels in introverts and extraverts: Effects on arousal and performance, J Pers Soc Psychol 46(6) (1987) 1303-1312.

[7] S.Y. Georgiev, C.V Christov, D.T Philipova, Ambiversion as independent personality characteristic, Activitas Nervosa Superior Rediviva 56(3-4) (2014) 65-72.

[8] D. Cohen, J.P. Schmidt, Ambiversion: characteristics of midrange responders on the Introversion-Extraversion continuum, J Pers Assess 43(5) (1979) 514-516.

[9] M. Crowe, R. Andel, N.L. Pedersen, L. Fratiglioni, M. Gatz, Personality and risk of cognitive impairment 25 years later, Psychol Aging 21(3) (2006) 573.

[10] C. Stough, J. Brebner, T. Nettelbeck, C. Cooper, T. Bates, G. Mangan, The relationship between intelligence, personality and inspection time, Brit J Psychol 87(2) (1996) 255-268.

[11] C. Doucet, R.M. Stelmack, An event-related potential analysis of extraversion and individual differences in cognitive processing speed and response execution, J Pers Soc Psychol 78(5) (2000) 956-964.

[12] D.L. Robinson, The neurophysiological bases of high IQ, Int J Neurosci 46(3-4) (1989) 209-234

[13] R.M. Stelmack, E. Achorn, A. Michaud, Extraversion and individual differences in auditory evoked response, Psychophysiol 14(4) (1977) 368-374. 
[14] E. Donchin, M.G. Coles, Is the P300 component a manifestation of [41] context updating, Behav Brain Sci 11(03) (1988) 357-374.

[15] J. Polich, Updating P300: an integrative theory of P3a and P3b, Clin Neurophysiol, 118(10) (2007) 2128-2148.

[16] J. Polich, J.R. Criado, Neuropsychology and neuropharmacology of P3a and P3b, Int J Psychophysiol 60(2) (2006) 172-185.

[17] M.D. Rugg, M.G. Coles, Electrophysiology of mind: Event-related brain potentials and cognition, Oxford University Press, England, 1995 .

[18] B. Brocke, K.G. Tasche, A. Beauducel, Biopsychological foundations of extraversion: differential effort reactivity and the differential P300 effect, Pers Indiv Differ 21(5) (1996) 727-738.

[19] G.M. Ditraglia, J. Polich J, P300 and introverted/extraverted personality types, Psychophysiol 28(2) (1991) 177-184.

[20] V. De Pascalis, On the psychophysiology of extraversion, In R. M. Stelmack (Ed), On the psychobiology of personality: Essays in hornor of Marvin Zuckerman, Elsevier Science, New York, 2004. 295327.

A. Beauducel, B. Brocke, A. Leue, Energetical bases of extraversion: Effort, arousal, EEG, and performance, Int J Psychophysiol 62(2) (2006) 212-223.

[21] R.J. Gurrera, B.F. O’Donnell, P.G. Nestor, J. Gainski, R.W. McCarley, The P3 auditory event-related brain potential indexes major personality traits, Biol Psychiat 49(11) (2001) 922-929.

[22] R.J Gurrera, D.F. Salisbury, B.F. O'Donnell, P.G. Nestor, R.W McCarley, Auditory P3 indexes personality traits and cognitive function in healthy men and women, Psychiatry Res 133(2-3) (2005) 215-228.

[23] D.T Philipova, Changes in N1 and P3 components of the auditory event-related potentials in extroverts and introverts depending on the type of the task, Folia medica 50(2) (2008) 24-31.

[24] M. Lindín, M. Zurrón, F. Díaz, Influences of introverted/extraverted personality types on P300 amplitude across repeated stimulation, J Psychophysiol 21(2) (2007) 75-82.

[25] T. Ortiz, V. Maojo, Comparison of the P300 wave in introverts and extraverts, Pers Indiv Differ 15(1) (1993) 109-112.

[26] C. Doucet, R.M. Stelmack, Movement time differentiates extraverts from introverts, Pers Indiv Differ 23(5) (1997) 775-786.

[27] S.T. Vorkapic, M. Tadinac, J. Rudez, P300 and extraversion in the visual oddball paradigm, Studia Psychologica 52(1) (2010) 3-14.

[28] L.E. Cox-Fuenzalida, A. Angie, S. Holloway, L. Sohl, Extraversion and task performance: A fresh look through the workload history lens, J Res Pers 40(4) (2006) 432-439.

[29] N. Yusoff, A.A Adamu, T. Begum, F. Reza, Amplitude and latency of P300 component from auditory stimulus in different types of personality: An event related potential study, Int J Med, Health, Biomed, Bioengineering Pharm Engineering 10(4) (2016) 184-187.

[30] M. Yusoff, A. Rahim, A. Esa, The Manual of USM Personality Inventory (USMaP-i). Malaysia: KKMED Publications, Malaysia, 2010.

[31] G.F. Woodman, A brief introduction to the use of event related potentials (ERPs) in studies of perception and attention, Atten Percept Psycho 72(8) (2010) 1-16.

[32] J.L. Andreassi, Psychophysiology: Human behaviour and physiological response (5th ed.), Lawrence Erlbaum Associates, London, 2007.

[33] E. Donchin, Surprise!...Suprise, Psychophysiology 18(5) (1981) 493-513.

[34] G. Sternberg, Extraversion and the P300 in a visual classification task, Pers Indiv Differ 16 (1994) 543-560.

[35] E. Courchesne, S.A. Hillyard, R. Galambos, Stimulus novelty, task relevance and the visual evoked potential in man, Electroencephalogr Clin Neurophysiol 39(2) (1975) 131-143.

[36] D. Friedman, G.V. Simpson, ERP amplitude and scalp distribution to target and novel events: effects of temporal order in young, middle-aged and older adults, Cognitive Brain Res 2(1) (1994) 49-63.

[37] D. Kahneman, Attention and effort, Prentice-Hall, New Jersey, 1973.

[38] J.D. Eastwood, A. Frischen, M.J. Fenske, D. Smilek, The unengaged mind: Defining boredom in terms of attention, Pers Psychol Sci 7(5) (2012) 482-495.

[39] B. Reimer, B. Mehler, The impact of cognitive workload on physiological arousal in young adult drivers: A field study and simulation validation, Ergonomics 54(10) (2011) 932-942.

[40] J. Von Gehlen, P. Sachse, Benefits of distraction, Soc Behav Pers 43(4) (2015) 601-612.
E. Barron, L.M. Riby, J. Greer, J. Smallwood, Absorbed in though: The effect of mind wandering on the processing of relevant and irrelevant events, Psychol Sci 22(5) (2011) 596-601.

[42] J. Smallwood, E. Beach, J.W. Schooler, T.C. Handy, Going AWOL in the brain: Mind wandering reduces cortical analysis of external events, J Cognitive Neurosci 20 (2008) 458-469.

[43] K. Christoff, A.M. Gordon, J. Smallwood, R. Smith, J.W. Schooler Experience sampling during fMRI reveals default network and executive system contributions to mind wandering, Proceedings of the National Academy of Sciences of the United States of America 106(21) (2009) 8719-8724.

[44] B. Mazoyer, L. Zago, E. Mellet et al., Cortical networks for working memory and executive functions sustain the conscious resting state in man, Brain Res Bull 54(3) (2001) 287-298.

J. Polich, A. Kok, Cognitive and biological determinants of P300: An integrative review, Biol Psychol 41(2) (1995) 103-146. 